\title{
Study of Selection of Timing System External Clock Synchronization Methods Based on Matrix Switch Technology
}

\author{
Wei $\mathrm{Wu}^{1, \mathrm{a}}$, Haidong Zou ${ }^{1, \mathrm{~b}^{*}}$ and Yupeng Zhang ${ }^{1, \mathrm{c}}$ \\ ${ }^{1}$ China Satellite Maritime Tracking and Control Department, Jiangsu, China \\ a448509294@qq.com, bcnflyfish@foxmail.com, ${ }^{c} 1648575334 @ q q . c o m$
}

Keywords: Timing system; Sync; variances; GPS; GLONASS; Beidou.

\begin{abstract}
The clock synchronization signal source of timing system is not equivalent to the receiving antenna. By using of matrix switch in the down link, and selecting the mean and variance characteristic parameters of the received signal, the performance of the work is evaluated. Using the corresponding software decision-making procedures, the optimum seeking method is automatic selected from the three signal demodulation boards. Experimental results show that the method has the merits of software automatically configuring, short switching time, overall better performance and so on.
\end{abstract}

\section{Introduction}

External clock synchronization of timing system is usually adopted to receive GPS signals, Beidou signal or GLONASS signal. In case of failure in selecting an input signal, manually switching to another signal is executed, and so forth. Typically, the system defaults to receive GPS signals, giving up the option of other two signals, even though which are both better than GPS in signal quality and timing accuracy. Literature studies[1-2] show that in some cases, using Beidou or GLONASS signal is no less than using GPS signal. Therefore, by using of matrix switch module in the circuit of three receivers, a method of software auto matching connections between the two antennae and the three receiver boards is proposed in this paper.

The research shows that the selection of performance measurements (usually depends on the model of sorting) is the focus of scholars at present. In the basis of researches and references, this paper building up state evaluation model of the timing system by matrix switch technology, combined with time series method [3], probability statistics in the variance principle [4-5]. And a type of GPS, Beidou, and GLONASS demodulation board is selected separately as samples for analysis, results proved the effectiveness of the research.

\section{Matrix Switch Technology}

Matrix switch is made up of a series of electronic switch arrays. Software controlled switching of the relay, which can form a certain topology of switches, to realize multiplex functions or to create switch matrix with multi-channel inputs and outputs $[6,7,8]$. For this system, there are two input signal and three output signal, that is, a matrix switch with 2-way RF (Radio Frequency) inputs and 3-way RF outputs is needed. By the appropriate program, its multiple inputs and outputs can automatic function. Its implementation is shown in Fig. 1. 


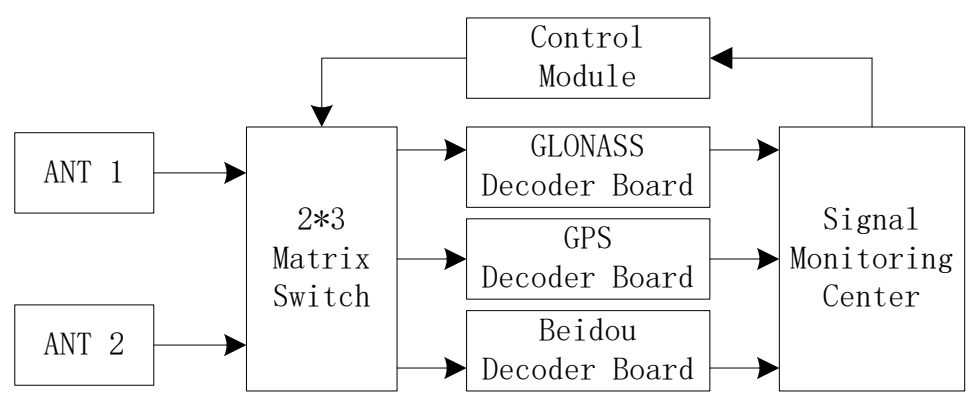

Figure 1. Diagram of hardware structure based on Matrix Switch

Fig. 1 shows that the two antennas links to the inputs of the RF matrix switch at the same time. The remote control module, through the corresponding signal selection algorithm, can finish its selection respectively with two in three timing signal decoder boards. Signal monitoring center is responsible for completing the timing resolution of two decoders, and determine whether the time-code flag is complete. If not, such decoder is not suitable for use, and the control unit should switch directly to the other decoder.

\section{Signal Selection Based on Variance}

Comparison of Characteristic Values. Given the signal quality, two elements should be taken into account. The first one is the received signal can smooth the time code decoding after passing through the corresponding decoders. This belongs to the qualitative indicators. Set the decoded signal as wi(n) $=0$ or $1, i=0,1,2$. and $n=0,1,2,3 \ldots$ The time code flag is decoded rightly when wi (n) equal to 1 , and is wrong when wi (n) equal to 0 . The second is the output signal amplitude of the decoder board following up the operational amplifier. By monitoring and comparing their average value and the flatness of the signal, that is variance of the signal. Set the monitor signal as ui (n), $i=1,1,2$. And $n=0$, $1,2,3 \ldots$, which denotes time series of signal. The expected value (mean amplitude) of monitor signals is:

$$
\overline{u_{i}}=\frac{1}{n} \sum_{k=0}^{n} u_{i}(k), i=0,1,2 .
$$

Generally speaking, the higher values of mathematical expectation, the greater the corresponding decoders in the received signal [9]. Then it's more beneficial for the stability of systems use, which means that the decoder works well. As to the variance:

$$
\begin{aligned}
& \mathrm{D}_{\mathrm{i}}=\frac{1}{n} \sum_{k=1}^{n}\left[u_{i}(k)-\bar{u}_{i}\right]^{2}=\frac{1}{n}\left(\sum_{k=1}^{n} u_{i}^{2}(k)-\sum_{k=1}^{n} \bar{u}_{i}^{2}\right) \\
& =\frac{1}{n} \sum_{k=1}^{n} u_{i}^{2}(k)-\bar{u}_{i}^{2}, i=0,1,2 .
\end{aligned}
$$

Variance is a measure of the deviation of actual measured values and expectation (average value) [10]. Therefore, smaller variance d denotes the time decoder works well. In order to more objectively reflect the actual working situation of receiver, variance D should be as small as possible.

Software Selection Process. As the amount of antennas is less than the demodulation boards, the program of selection is needed to ensure that there is a time code demodulation board in top working condition of the three ones at any one time. The process of the program is shown in Fig. 2. 


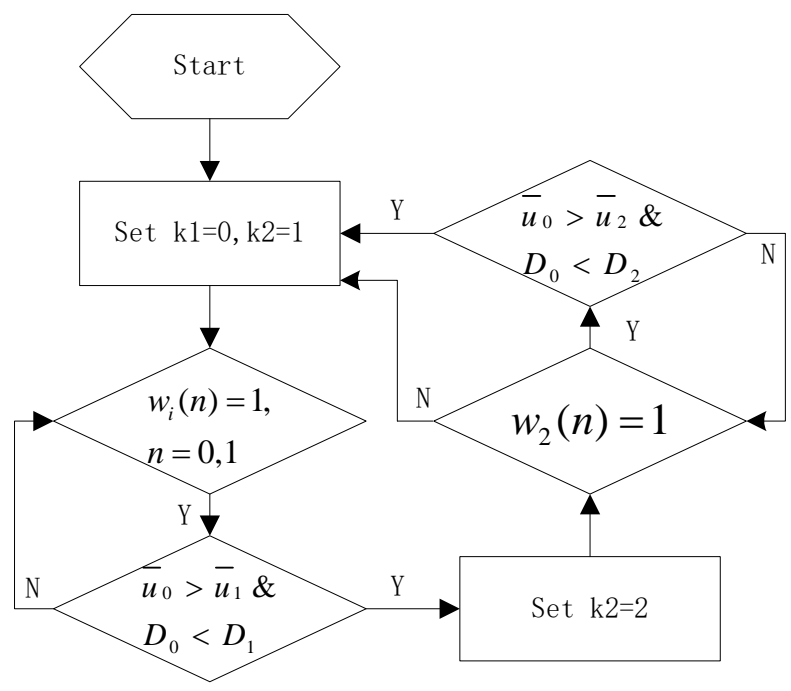

Figure 2. Diagram of signal selection

Fig. 2 shows that, after the system is powered on, first set the antenna 1 connect to the 1 st decoder board, and the antenna 2 connect to the 2 nd decoder board. Since then, the judgment of both time-code flag is normal, if it is, continue to judge both variance and signal amplitude value. If the 1 st decoder is significantly worse than the other one, then control module switches the antenna 1 to the 3rd decoder. Such judgments repeat again. Thus, a basic judgment process is complete. In addition, in view of work cycle of the system, the data collection period is set to 30 minutes, and the data is collected in every 5 minutes.

\section{Analysis of Measured Results}

In order to validate this program, three types of time decoder are selected and all the parameters of monitoring with all boards are recorded. Then, the variances of all the records are taken to choose the best one from which by calculations. Two cycles of measured signal value is shown in Table 1 .

Table 1 Signal levels of 3 Decoder Boards in 2 Time series (-dB)

\begin{tabular}{cccc}
\hline Period & No.0 & No.1 & No.2 \\
\hline & 78.2 & 79.2 & 137.1 \\
\multirow{2}{*}{1} & 78.5 & 79.6 & 137.9 \\
& 78.5 & 80.2 & 137.3 \\
& 78.9 & 81.1 & 138.2 \\
& 78.2 & 80.3 & 138.5 \\
& 79.8 & 80.1 & 138.1 \\
\hline & 79.6 & 137.5 & 78.5 \\
& 78.3 & 137.3 & 79.3 \\
& 78.6 & 138.3 & 79.8 \\
& 79.1 & 138.1 & 80.1 \\
& 79.6 & 138.9 & 80.6 \\
\hline
\end{tabular}

According to the data above, formulas (2-1) and (2-2) is used, and the results are shown as Table 2:

Table 2 Calculation vales of 3 Decoder Boards

\begin{tabular}{ccccccc}
\hline & $\overline{u_{0}}$ & $D_{0}$ & $\overline{u_{1}}$ & $D_{1}$ & $\overline{u_{2}}$ & $D_{2}$ \\
\hline 1 & -78.68 & 0.37 & -80.08 & 0.42 & -137.85 & 0.30 \\
2 & -79.12 & 0.31 & -137.98 & 0.39 & -79.83 & 0.69 \\
\hline
\end{tabular}


From the Table 2, in cycle 1, the parameter of demodulation board No.0 is obviously better than No.1. And in next cycle, software would automatically switch the matrix switch from No.1 to No.2. In the cycle 2, the No.0 demodulation board is still better than No.2, and in the next cycle, system still will switch demodulation board No.2 to No.1. Thus, the correctness of the method described in this paper is verified.

\section{Conclusions}

This paper focuses on the optimization problems of the time demodulation boards, which are not match with the receiver antennas in the number. Through the $2 \times 3 \mathrm{RF}$ matrix switch, antennas construct connections to three demodulation boards with little energy loss. On the software side, judgments are given under the qualitative and quantitative comparison of the received signal, and then best parameters when working of all the demodulation boards were calculated and selected.

However, fewer parameters were compared of all the selected items, up to a point, the selected time demodulation boards may not be the best ones. And, given the system stability considerations, there is some room for discussion of the length of the sampling period, these need to be continued in follow-up observations and improve, but this method put up in this paper is still providing a reference.

\section{References}

[1] Carine B. Comparing GPS-only with GPS+GLONASS positioning in a regional permanent GNSS network[J].GPS Solut,2007,11(2):97-106.

[2] ZHAI Yong, et al. Analysis of Positioning Precision Affected by Pseudo ranging Multipath for BDS [J].Journal of Navigation and Positioning, 2014, 7(2): 45-49.

[3] ZOU Haidong, et al. Research on the Prediction of Second Synchronization in Timing System using Cubic Exponential Smoothing[C].LEMCS,2015:1461-1464.

[4] YE L-E, HUANG X-X. A mean-variance optimization problem for continuous-time Markov decision processes [J]. Sci Sin Math, 2014, 44: 883-898.

[5] Guo X P. Constrained optimization for average cost continuous-time Markov decision processes [J]. IEEE Tans Automat Control, 2007, 52: 1139-1143.

[6] Xiang Xuezhi, Kai Xianglong, Zhang Zhenyu. Design of switching matrix module based on PXI bus [J].Foreign Electronic Measurement Technology, 2013, 12:76-79.

[7] WANG Yang, et al. A 0.5 um GaAs pHEMT RF Matrix Switch MMIC [J]. Microelectronics, 2013, 3: 329-332.

[8] SURKANTI P R, FURTH P M. Converting a threestage pseudo class-AB amplifier to a true-class-AB amplifier [J]. IEEE. Trans Cire Syst, 2012, 59(4):229-233.

[9] WANG Zheng, et al. Using SPSS Software Achieve Pharmacy Experiment Orthogonal Designs Variance Analysis [J]. Journal of Mathematical Medicine, 2014, 27(1):99-102.

[10] WANG Lulu, et al. New speech endpoint detection algorithm based on spectrum variance and spectral subtraction [J]. Computer Engineering and Applications, 2014, 50(8):194-197. 